\title{
Commission 3. (Notations, Unités et Economie des Publications)
}

I. La Commission recommande que la liste des notations publiée dans les Transactions of the I.A.U. 6, 345-55, I938, soit réimprimée et qu'un nombre suffisant d'exemplaires soit mis à la disposition des éditeurs des journaux astronomiques et des instituts astronomiques afin de les distribuer aux auteurs de rapports astronomiques.

2. Au cas où ce projet serait approuvé, la Commission recommande que la réimpression contienne une courte introduction annonçant que la Commission 3 a l'intention de revoir la liste des notations avant la prochaine Assemblée Générale et demandant que des critiques et des suggestions soient addressées au Président de la Commission 3.

\section{Commission 4. (EPHÉmÉRIDES)}

I. La Commission recommande que la désignation 'Temps Universel' (Universal Time; Weltzeit) soit seule utilisée par les astronomes pour désigner le temps solaire moyen, compté à partir de minuit du méridien de Greenwich.

Elle exprime le vœu que cette désignation remplace aussitôt que possible les autres expressions encore employées.

2. La Commission appuie très favorablement le projet formé par l'Observatoire National de Prague, de publier annuellement un Supplément international fournissant des données détaillées concernant les éclipses de lune et les occultations d'étoiles faibles pendant ces éclipses.

\section{Commission 5. (ANALYSE DE TRAVAUX ET BibliograPHIE)}

I. La Commission prend acte avec grand intérêt de ce que la Bibliographie Mensuelle de l'Astronomie reprend la publication de son bulletin mensuel en liaison avec le Bulletin Analytique du Centre National de la Recherche Scientifique (Paris) et envisage de combler la lacune due à la guerre.

La Commission estime que la subvention attribuée à la B.M.A. devrait être continuée à concurrence de I500 francs-or pour la période des trois années à venir (I949, I950 et I95I) et que la subvention prévue antérieurement et qui n'a pas encore été touchée, devrait être mise à la disposition de la B.M.A. pour aider à combler la lacune due à la guerre.

2. La Commission a appris également avec un vif intérêt que le Jahresbericht pour les années I94I et r942 a été publié et que le premier des deux volumes consacrés aux années I943-46 est imprimé; elle souhaite voir la publication du Jahresbericht se continuer régulièrement.

Elle recommande qu'un exemplaire des publications soit adressé dès parution à l'Astronomisches Rechen-Institut, ( 17 a) Heidelberg, Seminarienhaus, Augustinergasse I5, Baden, U.S. Zone, Allemagne, ainsi qu'un exemplaire des publications éditées au cours des années I943-46 et suivantes pour autant que cela n'ait pas été fait.

3. La Commission ayant pris connaissance de l'état d'avancement du travail entrepris pour combler la lacune entre la Bibliographie de Houzeau-Lancaster et le Jahresbericht, serait désireuse de voir le Comité National Belge prendre toutes mesures possibles pour mener ce travail à bien.

4. La Commission est heureuse d'apprendre que, suite à sa recommandation 4, prise lors du Congrès de Stockholm en I938, Monsieur A. N. Vyssotsky a terminé la traduction de la partie astronomique des chroniques russes médiévales, que le manuscrit en est prêt et que la publication en sera assurée par l'Observatoire de Lund.

5. La Commission, qui avait demandé par sa recommandation 5 prise lors du Congrès de Stockholm en I938 que l'Observatoire de Lund établisse un catalogue sur fiches des astronomes et des observatoires, estime qu'il serait fort utile de publier dès que possible une liste des observatoires et des astronomes, telle qu'elle avait été éditée antérieurement par l'Observatoire Royal de Belgique. 